\title{
Segurança do paciente: Percepção de profissionais de saúde de uma fundação pública estadual de Belém-PA
}

\author{
Patient safety: Perception of health professionals from a state public foundation of \\ Belém-PA
}

\author{
Seguridad del paciente: percepción de los profesionales sanitarios de una \\ Fundación pública estatal de Belém-PA
}

Fabiola Santana de Oliveira Costa ${ }^{1 *}$, Heliana Helena de Moura Nunes ${ }^{2}$, Marcelo Williams Oliveira de Souza ${ }^{3}$.

\section{RESUMO}

Objetivo: Apresentar o entendimento dos profissionais de saúde de uma Fundação Publica estadual, sobre os protocolos de segurança do paciente. Métodos: Estudo descritivo, exploratório, com abordagem quantitativa. Desenvolvido na Fundação Hospital de Clínicas Gaspar Vianna (FHCGV), Belém-PA. Os setores foram: Centro Cirúrgico (C.C) e Unidade Coronariana (UCA). A amostra foram profissionais de saúde da equipe multiprofissional (enfermeiros, médicos, fisioterapeutas, psicólogos, fonoaudiólogo, técnicos de enfermagem e auxiliares de enfermagem). A pesquisa foi realizada em três momentos: aplicado o instrumento de coleta de dados, analisados a totalidade dos formulários aplicados aos profissionais de saúde e realizado o estudo estatístico utilizando o Sistema Bioestat 5.0. Resultados: Caracterizam a população participante e aos aspectos relacionados ao entendimento dos profissionais sobre os protocolos de segurança do paciente. A população foi de 109 profissionais de saúde que atuam nos setores do estudo. Desse quantitativo apenas 80 profissionais de saúde $(73,4 \%)$ foram participantes da pesquisa, sendo 14 enfermeiros (17,5\%), 40 técnicos de enfermagem (50\%), 19 médicos (23,75\%), 05 fisioterapeutas $(6,25 \%), 01$ fonoaudiólogo $(1,25 \%)$ e 01 psicólogo (1,25\%). Foram excluídos 29 profissionais. Conclusão: Permitiu-se identificar a representatividade da equipe de enfermagem nos processos assistenciais, sendo a participação relevante para os resultados que demonstram quais protocolos estão mais sedimentados.

Palavras-chave: Segurança do paciente, Protocolos de segurança do paciente, Pessoal de saúde.

\begin{abstract}
Objective: To present the understanding of the health professionals of a state public foundation, on the patient safety protocols. Methods: This is a descriptive, exploratory study with a quantitative approach. Developed at the Hospital de Clínicas Gaspar Vianna Foundation (FHCGV), Belém-PA. The sectors were: surgical Center (c. c) and coronary unit (UCA). The sample was health professionals of the multidisciplinary team (nurses, physicians, physiotherapists, psychologists, speech-language pathologists, nursing technicians and nursing assistants). The research was carried out in three moments: applied the data collection instrument, analyzed the totality of the forms applied to health professionals and carried out the statistical study using the Bioestat 5.0 system. Results: They characterize the participant population and the aspects related to the professionals
\end{abstract}

${ }_{1}^{1}$ Fundação Pública Estadual Hospital de Clínicas Gaspar Viana (FHCGV), Belém-Pará.

*E-mail: fabiolacosta.enf@gmail.com

2 Fundação Santa Casa de Misericórdia do Pará (FSCMP), Belém-PA.

${ }^{3}$ Universidade do Estado do Pará (UEPA), Belém-PA. 
' understanding of the patient's safety protocols. The population was 109 health professionals working in the study sectors. of this quantitative only 80 health professionals $(73.4 \%)$ were participants in the study, 14 nurses (17.5\%), 40 nursing Technicians (50\%), 19 physicians (23.75\%), 05 physiotherapists $(6.25 \%)$, 01 speech therapist (1.25\%) and 01 psychologist (1.25\%). We excluded 29 professionals. Conclusion: It was possible to identify the representativeness of the nursing team in the care processes, being the relevant participation for the results that demonstrate which protocols are more sedimentary.

Key words: Patient safety, Patient safety protocols, Health personnel.

\section{RESUMEN}

Objetivo: presentar la comprensión de los profesionales sanitarios de una Fundación pública estatal, sobre los protocolos de seguridad del paciente. Métodos: se trata de un estudio descriptivo y exploratorio con un enfoque cuantitativo. Desarrollado en la Fundación Hospital de clínicas Gaspar Vianna (FHCGV), Belém-PA. Los sectores fueron: Centro quirúrgico (c. c) y unidad coronaria (UCA). La muestra era profesionales de la salud del equipo multidisciplinar (enfermeras, médicos, fisioterapeutas, psicólogos, patólogos del hablalenguaje, técnicos de enfermería y asistentes de enfermería). La investigación se llevó a cabo en tres momentos: aplicó el instrumento de recolección de datos, analizó la totalidad de las formas aplicadas a los profesionales de la salud y realizó el estudio estadístico utilizando el sistema Bioestat 5,0. Resultados: caracterizan a la población participante y a los aspectos relacionados con la comprensión por los profesionales de los protocolos de seguridad del paciente. La población era de 109 profesionales sanitarios que trabajaban en los sectores de estudio. de este cuantitativo sólo 80 profesionales de la salud $(73,4 \%)$ fueron participantes en el estudio, 14 enfermeras (17,5\%), 40 técnicos de enfermería $(50 \%), 19$ médicos $(23,75 \%), 05$ fisioterapeutas (6,25\%), 01 Logopedia $(1,25 \%)$ y 01 psicólogo $(1,25 \%)$. Excluimos a 29 profesionales. Conclusión: fue posible identificar la representatividad del equipo de enfermería en los procesos de atención, siendo la participación relevante para los resultados que demuestran qué protocolos son más sedimentarios.

Palabras clave: Seguridad del paciente, Protocolos de seguridad del paciente, Personal sanitario.

\section{INTRODUÇÃO}

A Segurança do Paciente tem ganhado força nas instituições de saúde por ser tema debatido mundialmente nas últimas décadas, tornando-se subvenção essencial para o progresso da qualidade dos serviços de saúde. O debate da temática foi fortalecido em 1999, a partir da publicação do Instituto de Medicina dos Estados Unidos (IOM) intitulada Errar é Humano: Construindo um Sistema de Saúde Mais Seguro [To Err is Human: Building a Safer Health System] (INSTITUTE OF MEDICINE, 1999). A Segurança do Paciente já faz parte da agenda política desde a mobilização do Ministério da Saúde junto à Organização Mundial da Saúde (OMS), com o seu ápice em 2013, a partir da publicação da Portaria № 520/2013 que institui o Programa Nacional de Segurança do Paciente no Brasil (BRASIL, 2013).

São crescentes as iniciativas para a promoção da segurança e da qualidade na assistência à saúde em âmbito mundial, com envolvimento da alta direção das instituições até seus colaboradores. Como consequência, a meta de qualidade nos diversos serviços oferecidos à sociedade implica a otimização dos resultados (OLIVEIRA JR, et., 2013).

A prestação do cuidado de saúde é uma atividade de grande complexidade ao nível do paciente tratado e em nível de financiadores, que constituem os atores da saúde. Uma das características dos serviços de saúde é atender à necessidade dos usuários através da produção de resultados, porém esses muitas vezes são 
incertos e expressos em erros e complicações, com custos elevados e crescente insatisfação da população tratada. Nesse contexto, surge a necessidade dos serviços de saúde adotar medidas para reduzir os erros, através da adoção de diretrizes clínicas e terapêuticas para obtenção de resultados mais satisfatórios. No entanto, por ser a saúde um sistema complexo e dinâmico composto por diversos agentes (médicos, enfermeiros, pacientes, gestores...) e que comumente apresentam interesses conflitantes, as organizações de saúde estarão sempre mais vulneráveis a erros e resistentes à mudança para melhor (SOUSA P e MENDES W, 2014b).

Em análise, sobre qualidade do cuidado e sua relação com outras temáticas no campo da avaliação de serviços de saúde, emergem preocupações voltadas para as pressões relacionadas ao alto custo do cuidado em saúde, a incorporação tecnológica, ao aumento da carga de trabalho dos profissionais de saúde e ao envelhecimento da população. Despertando, preocupação com os processos de qualidade desenvolvidos nas organizações de saúde, com ênfase em ações direcionadas à melhoria contínua e à prestação de contas (FISHER ES, et al., 2010; WENNBERG JE, 2010).

Embora, os hospitais sejam campo de produção de conhecimento para o desenvolvimento do Sistema de Saúde em diversas áreas, como a de referência assistencial de alta complexidade, polos formadores de recursos humanos, desenvolvimento de pesquisas e incorporação de novas tecnologias que colaborem para melhoria da população. A adequação as práticas de ensino, também representam melhoria na formação dos profissionais e consequentemente aos serviços que os mesmos estarão inseridos (BARATA LRB, et al., 2010).

No decorrer dos últimos anos tem-se observado que a segurança do paciente tomou grandes repercussões no meio científico e assistencial, uma vez que as ocorrências de eventos adversos vêm aumentando e tornando-se cada vez mais alvo de processos judiciais, o que ocasiona aumento de custos indenizatórios para as organizações de saúde e algumas vezes para os profissionais de saúde. Para Lima FDMA (2014) é fato que os cuidados de saúde prestados de forma insegura vêm contribuindo para o aumento da morbimortalidade, já que muitos erros poderiam ser evitados através da implantação de programas de qualidade e adoção de meios de monitoramento dos resultados ao paciente hospitalizado. A segurança do paciente é definida como a diminuição, a um mínimo permissível, do risco de dano associado à saúde, desnecessário (ANVISA, 2014).

O cuidado seguro resulta tanto de ações corretas dos profissionais de saúde, como de processos e sistemas adequados nas instituições e serviços, assim como de políticas governamentais regulatórias, exigindo um esforço coordenado e permanente (REBRAENSP, 2013). Portanto, este estudo objetivou apresentar o entendimento dos profissionais de saúde de uma Fundação Publica estadual, sobre os protocolos de segurança do paciente.

\section{MÉTODOS}

Este estudo é do tipo descritivo, exploratório, com abordagem quantitativa. A pesquisa foi desenvolvida na Fundação Hospital de Clínicas Gaspar Vianna (FHCGV), Belém-PA. Os setores selecionados para realização da pesquisa foram o Centro Cirúrgico (C.C) e Unidade Coronariana (UCA) pelo fato desses serviços terem 
sido selecionados pelo Núcleo de Segurança do Paciente para implantação de projetos piloto dos protocolos de segurança do paciente.

A população da pesquisa foram os profissionais de saúde da equipe multiprofissional (enfermeiros, médicos, fisioterapeutas, psicólogos, fonoaudiólogo, técnicos de enfermagem e auxiliares de enfermagem) que atuam na FHCGV, nos setores selecionados para o estudo, com amostra de 80 profissionais. Como critério de inclusão foi utilizado ser profissional da área da saúde; pertencer ao quadro de pessoal dos setores selecionados; estar atuando no setor a pelo menos um ano; ter carga horária de trabalho mínima de 20h semanais.

Enquanto que, os critérios de exclusão foram pertencer à empresa terceirizada ou ser residente, acadêmico e estagiário, encontrar-se afastado do setor no período da coleta e desenvolver jornada inferior a $20 \mathrm{~h}$ semanais. A coleta de dados ocorreu no período de agosto a novembro de 2016, após autorização das instituições participantes e aprovação do projeto pelo Comitê de Ética em Pesquisa (CEP) da Fundação Hospital de Clínicas Gaspar Vianna e da Fundação Santa Casa de Misericórdia do Pará.

No primeiro momento, foi aplicado o instrumento de coleta de dados sendo um formulário semiestruturado contendo perguntas fechadas em formato Microsoft Word. O formulário apresentou-se dividido em três tópicos. O primeiro tópico abrange a caracterização dos participantes com 05 perguntas, o segundo tópico é referente aos aspectos relacionados ao entendimento dos protocolos de segurança do paciente com 12 indagações e o terceiro tópico está relacionado às dificuldades para notificação de incidentes e eventos adversos e abrange 06 perguntas. No segundo momento, foram analisados a totalidade dos formulários aplicados aos profissionais de saúde, participantes da pesquisa, no período de agosto a novembro de 2016, em seguida foram tabulados em planilha do Microsoft Excel.

No terceiro momento, foi realizado o estudo estatístico utilizando o Sistema Bioestat 5.0, sendo o grau de significância adotado de $p<0,05$, sendo os dados apresentados em tabelas e gráficos e analisados de forma descritiva por distribuição de frequência das variáveis com maior relevância.

O projeto de pesquisa foi submetido à Plataforma Brasil, e, em seguida, aprovado pelo Comitê de Ética em Pesquisa (CEP) com número de parecer 1.571.630 e CAAE: 54145216.7.0000.517. Os pesquisadores comprometeram-se com as normas preconizadas pela Resolução do CNS 466/12 e suas complementares assegurando que nenhum participante foi submetido aos instrumentos de coleta de dados sem receber as devidas orientações e sem assinar o Termo de Consentimento Livre e Esclarecido.

\section{RESULTADOS}

Os resultados descritos abaixo se referem aos dados pessoais que caracterizam a população participante da pesquisa e aos aspectos relacionados ao entendimento que esses profissionais de saúde da FHCGV têm sobre os protocolos de segurança do paciente. A amostra estimada foi de 109 profissionais, da equipe multiprofissional, que atuam nos setores de centro cirúrgico (C.C) e unidade coronariana (UCA).

Desse quantitativo apenas 80 profissionais de saúde $(73,4 \%)$ da população estimada, foram participantes da pesquisa, sendo 14 enfermeiros (17,5\%), 40 técnicos de enfermagem (50\%), 19 médicos (23,75\%), 05 fisioterapeutas (6,25\%), 01 fonoaudiólogo (1,25\%) e 01 psicólogo (1,25\%). Foram excluídos 29 profissionais, 
sendo que 28 pelos critérios de exclusão e 01 por não está de acordo com a participação na pesquisa. O que corresponde a $26,60 \%$ de não participantes da população real.

Os dados descritos na Tabela 1 correspondem à caracterização da amostra pesquisada. Enquanto, que a tabulação dos resultados referentes aos aspectos relacionados ao entendimento sobre os protocolos de segurança do paciente está representada nas Tabelas de 2 a 5.

Tabela 1 - Distribuição dos entrevistados segundo variáveis do Perfil Profissional, n=80. Belém-PA, 2017.

\begin{tabular}{llcc}
\hline Variáveis & $\mathbf{N}$ & $\%$ & $\boldsymbol{P}^{\boldsymbol{*}}$ \\
\hline 1) Sexo* & 52 & 65,00 & \\
Masculino & 28 & 35,00 & 0,0037 \\
Feminino & 28 & \\
\hline
\end{tabular}

\section{2) Categoria Profissional*}

$\begin{array}{lcc}\text { Téc. de Enfermagem } & 40 & 50,00 \\ \text { Médico } & 19 & 23,75 \\ \text { Enfermeiro } & 14 & 17,5 \\ \text { Fisioterapeuta } & 5 & 6,25 \\ \text { Psicólogo } & 1 & 1,25 \\ \text { Fisioterapeuta } & 5 & 6,25 \\ \text { Fonoaudiólogo } & 1 & 1,25\end{array}$

\section{3) Formação Acadêmica*}

$\begin{array}{lccc}\text { Nível Médio } & 27 & 33,75 & 0,0004 \\ \text { Graduação } & 13 & 16,25 & \\ \text { Especialização } & 31 & 38,75 & \\ \text { Mestrado } & 8 & 10,00 & \\ \text { Doutorado } & 1 & 1,25 & \end{array}$

\section{4) Tempo de atuação em serviço de saúde*}

\begin{tabular}{lccc}
\hline Menor que 5 anos & 6 & 7,50 & \\
Entre 5 e 10 anos & 18 & 22,50 & \\
Maior que 10 anos & 56 & 70,00 & $<0.0001$
\end{tabular}

\section{5) Setor de lotação*}

Centro Cirúrgico

Unidade Coronariana

$39 \quad 48,75$

$41 \quad 51,25$

0,9110

\begin{tabular}{cc}
\hline Total $\mathbf{N}$ & $\mathbf{8 0}$ \\
\hline Fonte: Dados da Pesquisa, 2017. & Teste do Qui-Quadrado com $\alpha=0,05$
\end{tabular}

$\mathrm{Na}$ tabela acima estão representados os dados referentes à distribuição dos participantes segundo variáveis categóricas do perfil profissional. Observa -se que a população estudada, foi predominantemente do gênero masculino, o que corresponde a $65 \%$ da amostra total. Em relação à categoria profissional, os técnicos de enfermagem apresentaram a maior expressividade no estudo com $50 \%$ de participação, seguido da categoria de médicos com $23,75 \%$. 
Quanto aos dados referentes aos perfis dos participantes do estudo, conforme a formação acadêmica foi observado que a titulação de especialista obteve a maior frequência, com $38,75 \%$ dos participantes. Enquanto que, 33,75\% apresentam apenas a formação de nível médio e corresponde a categoria profissional de técnico de enfermagem.

O tempo de atuação dos profissionais em serviços de saúde obteve a maior frequência no intervalo superior a 10 anos, com expressividade de 56 profissionais participantes do estudo, o que corresponde a $70 \%$ da amostra total. Outro dado, é que apenas 7,5\% informaram tempo inferior há 5 anos, caracterizando uma população madura quanto ao tempo de atuação profissional.

Em relação ao setor de lotação dos profissionais, a maior amostragem corresponde ao setor de Unidade Coronariana (UCA). Destaca-se, que do percentual total de profissionais dos setores selecionados, os técnicos de enfermagem e enfermeiros representam o maior percentual de $67,5 \%$, o que aponta uma significante representatividade da equipe de enfermagem no estudo.

Tabela 2 - Distribuição dos resultados quanto ao conhecimento, dos profissionais, de quais são os protocolos de segurança do paciente, Belém-PA, 2017.

\begin{tabular}{|c|c|c|c|c|c|c|c|c|c|}
\hline \multirow[b]{3}{*}{ Profissional } & \multirow{2}{*}{\multicolumn{2}{|c|}{1 - Totalmente }} & \multirow{2}{*}{\multicolumn{2}{|c|}{$\begin{array}{l}2 \text { - Quase } \\
\text { Totalmente }\end{array}$}} & \multirow{2}{*}{\multicolumn{2}{|c|}{$\begin{array}{l}\text { 3-Quase } \\
\text { Nenhum }\end{array}$}} & \multirow{2}{*}{\multicolumn{2}{|c|}{4 - Nenhum }} & \multirow[b]{3}{*}{ Tota } \\
\hline & & & & & & & & & \\
\hline & № & $\%$ & № & $\%$ & № & $\%$ & № & $\%$ & \\
\hline Enfermeiro & 13 & 92,86 & 1 & 7,14 & & 0,00 & 0 & 0,00 & 14 \\
\hline Fisioterapeuta & 1 & 20,00 & 4 & 80,00 & & 0,00 & 0 & 0,00 & 5 \\
\hline Fonoaudiólogo & 1 & 100,0 & & 0,00 & & 0,00 & 0 & 0,00 & 1 \\
\hline Médico & 4 & 21,05 & 10 & 52,63 & 5 & 26,32 & 0 & 0,00 & 19 \\
\hline Psicólogo & 0 & 0,00 & 1 & 100,00 & & 0,00 & 0 & 0,00 & 1 \\
\hline Téc. Enfermagem & 26 & 65,00 & 12 & 30,00 & 2 & 5,00 & 0 & 0,00 & 40 \\
\hline Total geral & 45 & 56,25 & 28 & 35,00 & 7 & 8,75 & 0 & 0,00 & 80 \\
\hline
\end{tabular}

Fonte: Dados da Pesquisa, 2017.

No estudo, os dados obtidos na tabela, estão relacionadas as respostas conforme a categoria profissional dos participantes. Apresenta um percentual de 56,25\%, da amostra geral, de respostas positivas do reconhecimento de quais são os protocolos de segurança do paciente em sua totalidade, mostrando uma área neutra com tendência positiva (35\%) ao conhecimento de quase totalidade sobre o assunto.

Outro dado relevante é que dentre os pesquisados, os profissionais de enfermagem representaram a maior amostragem do conhecimento sobre a totalidade dos protocolos. Enquanto, que a categoria médica demonstrou um percentual de $26.32 \%$ (Quase Nenhum) de tendência negativa, do conhecimento sobre o assunto. 
Tabela 3 - Distribuição sobre a opinião dos profissionais quanto à utilização dos protocolos de segurança do paciente na FHCGV, Belém-PA, 2017.

\begin{tabular}{|c|c|c|c|c|c|c|c|c|c|}
\hline \multirow[b]{2}{*}{ Profissional } & \multicolumn{2}{|c|}{1 - Totalmente } & \multicolumn{2}{|c|}{$\begin{array}{l}2 \text { - Quase } \\
\text { Totalmente }\end{array}$} & \multicolumn{2}{|c|}{$\begin{array}{l}\text { 3-Quase } \\
\text { Nenhum }\end{array}$} & \multicolumn{2}{|c|}{ 4-Nenhum } & \multirow[b]{2}{*}{ Total } \\
\hline & № & $\%$ & № & $\%$ & № & $\%$ & № & $\%$ & \\
\hline Enfermeiro & 4 & 28,57 & 10 & 71,43 & & 0,00 & 0 & 0,00 & 14 \\
\hline Fisioterapeuta & 4 & 80,00 & 1 & 20,00 & & 0,00 & 0 & 0,00 & 5 \\
\hline Fonoaudiólogo & & 0,00 & 1 & 100,00 & & 0,00 & 0 & 0,00 & 1 \\
\hline Médico & 4 & 21,05 & 12 & 63,16 & 3 & 15,9 & 0 & 0,00 & 19 \\
\hline Psicólogo & 1 & 100,0 & & 0,00 & & 0,00 & 0 & 0,00 & 1 \\
\hline Téc. Enfermagem & 24 & 60,00 & 14 & 35,00 & 2 & 5,00 & 0 & 0,00 & 40 \\
\hline Total geral & 37 & 46,25 & 38 & 47,50 & 5 & 6,25 & 0 & 0,00 & 80 \\
\hline
\end{tabular}

Fonte: Dados da Pesquisa, 2017.

Observa-se na Tabela 3 que dos profissionais investigados um percentual expressivo de $46,25 \%$ relata que a FHCGV, utiliza os protocolos de segurança totalmente e $47,5 \%$ informa ser parcialmente. Outro aspecto relevante é que a opção de respostas (nenhum) não foi mencionada pelos profissionais.

Tabela 4 - Distribuição dos resultados quanto a opinião, dos profissionais de saúde, se a utilização dos protocolos de segurança do paciente, tornam as práticas assistenciais mais seguras, Belém-PA, 2017.

$$
\begin{array}{llll}
1 \text { 1-Totalmente } & 2 \text {-Quase } & 3 \text {-Quase } & 4-\text { Nenhum } \\
& \text { Totalmente } & \text { Nenhum } &
\end{array}
$$

\begin{tabular}{lccccccccc}
\hline Profissional & № & $\%$ & № & $\%$ & № & $\%$ & $\mathbf{N}$ & $\%$ & Total \\
\hline Enfermeiro & 12 & 85,71 & 2 & 14,9 & 0 & 0,00 & 0 & 0,00 & 14 \\
Fisioterapeuta & 3 & 60,00 & 2 & 40,0 & 0 & 0,00 & 0 & 0,00 & 5 \\
Fonoaudiólogo & 1 & 100,0 & 0 & 0,00 & 0 & 0,00 & 0 & 0,00 & 1 \\
Médico & 15 & 78,95 & 3 & 15,9 & 0 & 0,00 & 1 & 5,26 & 19 \\
Psicólogo & 1 & 100,0 & 0 & 0,00 & 0 & 0,00 & 0 & 0,00 & 1 \\
Téc. Enfermagem & 32 & 80,00 & 8 & 20,0 & 0 & 0,00 & 0 & 0,00 & 40 \\
& & & & 0 & & & & & \\
\hline Total geral & $\mathbf{6 4}$ & $\mathbf{8 0 , 0 0}$ & $\mathbf{1 5}$ & $\mathbf{1 8 , 5}$ & $\mathbf{0}$ & $\mathbf{0 , 0 0}$ & $\mathbf{1}$ & $\mathbf{1 , 2 5}$ & $\mathbf{8 0}$ \\
\hline
\end{tabular}

Fonte: Dados da Pesquisa, 2017.

A tabela 4 demonstra a opinião dos profissionais de saúde se a utilização dos protocolos de segurança do paciente torna as práticas assistenciais mais seguras. Os resultados obtidos mostram que $80 \%$ concordam (Totalmente) e 18,75\% (Quase Totalmente), apresentando uma forte tendência de boa percepção geral sobre a segurança do paciente no âmbito institucional. 
Tabela 5 - Distribuição da importância atribuída aos protocolos de segurança no âmbito institucional, BelémPA, 2017.

\begin{tabular}{llll}
\hline 1 - Totalmente & 2 -Quase & 3-Quase & $4-$ Nenhum \\
& Totalmente & Nenhum &
\end{tabular}

\begin{tabular}{|c|c|c|c|c|c|c|c|c|c|}
\hline Profissional & № & $\%$ & № & $\%$ & № & $\%$ & № & $\%$ & Total \\
\hline \multirow[t]{2}{*}{ Enfermeiro } & 8 & 57,14 & 4 & 28,7 & 2 & 14,2 & & 0,00 & 14 \\
\hline & & & & & & 9 & & & \\
\hline Fisioterapeuta & 5 & 100,00 & & 0,00 & & 0,00 & & 0,00 & 5 \\
\hline Fonoaudiólogo & 1 & 100,00 & & 0,00 & & 0,00 & & 0,00 & 1 \\
\hline Médico & 11 & 57,89 & 6 & 31,8 & 1 & 5,26 & 1 & 5,26 & 19 \\
\hline Psicólogo & 1 & 100,00 & & 0,00 & & 0,00 & & 0,00 & 1 \\
\hline Téc. Enfermagem & 27 & 71,05 & 9 & 23,8 & 2 & 5,26 & & 0,00 & 38 \\
\hline \multirow[t]{2}{*}{ Total geral } & 53 & 67,95 & 19 & 24,3 & 5 & 6,41 & 1 & 1,28 & 78 \\
\hline & & & & 6 & & & & & \\
\hline
\end{tabular}

Fonte: Dados da Pesquisa, 2017.

Os resultados obtidos apresentam um percentual positivo de $67,95 \%$ quanto ao grau de importância que a FHCGV atribui aos protocolos de segurança do paciente.

\section{DISCUSSÃO}

A partir da análise dos resultados obtidos dos profissionais de saúde da FHCGV, sobre as questões que permeia a segurança do paciente no âmbito hospitalar e suas interferências no processo assistencial. Foi identificado que o conhecimento entre as categorias profissionais pertencentes às mesmas unidades de trabalho é divergente, assim como, as dificuldades percebidas intersetorialmente se diferenciam.

No entanto, os aspectos relacionados ao âmbito institucional que revelam a percepção dos profissionais de como essa organização de saúde se modelam para atender e disseminar o Programa Nacional de Segurança do Paciente, de forma transversal e interdisciplinar, por meios da divulgação de conteúdos que visem à incorporação pela equipe assistencial de práticas mais seguras no seu cotidiano de trabalho. Foi observada uma tendência positiva, com um percentual de $47,5 \%$ de quase totalidade e de $46,25 \%$ de totalidade, com destaque para os enfermeiros e médicos, quanto à utilização dos protocolos de segurança do paciente na FHCGV.

Esses dados retratam que a instituição do estudo, adota o programa nacional de segurança do paciente como estratégia de gestão, mas ainda se encontra em processo de maturidade da cultura de segurança, com característica atual para a tendência do tipo "calculada".

Para Hudson P (2003), a cultura calculada é gerenciada, mas as abordagens são ainda muito equilibradas, com sistema de gerenciamento/ gestão de risco e foco na exação de dados. Para Mendes Junior WV (2012) a cultura organizacional influencia o sentido por meio do qual os profissionais aprendem e compartem o conhecimento. 
Nesse sentido, a comunicação entre os profissionais, os gestores e os clientes, configuram-se em peças chaves para o estabelecimento de uma política de transversalidade e assim, a comunicação torna-se um elemento essencial no cuidado (BROCA PV e FERREIRA MA, 2012).

Percebe-se que apesar das organizações de saúde terem características semelhantes, pela natureza do trabalho, existe um longo caminho a percorrer em busca da "fiabilidade/confiabilidade", já que a probabilidade de erros ou incidentes é real, mas existem meios que permitem a minimização do seu impacto e consequentemente, melhores resultados (SOUSA P e MENDES W, 2014a).

Outro aspecto relevante é o conhecimento que os profissionais de saúde têm acerca dos protocolos de segurança do paciente. Os dados coletados apontam para um percentual de $56,25 \%$ de respostas afirmativas e com destaque para os profissionais da equipe de enfermagem. Dentre os protocolos investigados os que obtiveram um maior percentual de confirmação, quanto à implantação nas unidades se serviço, foram o "Protocolo de Identificação do Paciente e Protocolo de Higienização das mãos", com um percentual de 82,5\% e $76,25 \%$, respectivamente, de totalidade nas respostas, reforçando que ainda se encontra em processo de implementação na FHCGV, os demais protocolos estabelecidos pelo Ministério da Saúde.

A Portaria MS/GM n 529/2013, estabelece um conjunto de protocolos básicos, definidos pela OMS, que constituem instrumentos para construir uma prática assistencial segura e são componentes obrigatórios dos Planos de Segurança do Paciente (PSP) desenvolvidos pelos Núcleos de Segurança do Paciente (NSP), dos estabelecimentos de saúde, seguindo as diretrizes definidas nas legislações, porém compatíveis com a realidade de cada serviço e com metas a serem atingidas (BRASIL, 2014).

Embora os protocolos estabelecidos pelo Ministério da Saúde/Agencia Nacional de Vigilância Sanitária, descrevam cuidados básicos para segurança do paciente. Sabe-se que a implementação nos locais de prestação de serviço perpassa pela cultura organizacional e a forma como as equipes se organizam no seu ambiente de trabalho (COREN-SP, 2010).

Os NSP devem, antes de tudo, atuar como articuladores e incentivadores das demais instâncias do hospital que gerenciam os risco s e ações de qualidade, promovendo complementariedade de sinergias neste âmbito (BRASIL, 2014). Nessa lógica a instituição através do NSP e demais serviços estratégicos da organização, vem desenvolvendo ações de forma gradativa com intuito de implementar processos que contemplem a segurança do paciente.

Os resultados encontrados na pesquisa apontam que a maioria dos profissionais de saúde reconhecem a relevância da utilização dos protocolos estabelecidos pela OMS, como métodos que propiciam práticas assistenciais mais seguras, como demonstram os resultados encontrados na TABELA 2.5. Assim como, informam positivamente a importância atribuída aos protocolos de segurança pela FHCGV, estando representado no percentual de $92,31 \%$ de tendência positiva das respostas obtidas, referente a questão.

É fundamental que cada hospital tenha artifícios próprios e criativos para garantir o envolvimento e a participação de seus colaboradores no que se refere às políticas institucionais e os resultados ao qual se pretende alcançar (NISHIO EA e FRANCO MT, 2011).

REAS/EJCH | Vol. 11 (9) | e350 | DOI: https://doi.org/10.25248/reas.e350.2019 Página 9 de 12 
A transdisciplinaridade tem sido apontada como único modo de se garantir a cultura e segurança do paciente nas instituições, pois traz a possibilidade de revisão do atual paradigma de ciência e, consequentemente, de saúde, revelando possibilidades de repensar a tarefa e o valor de um projeto coletivo de trabalho (FONSECA AS, et al., 2014).

Atualmente, se discute que os erros e as falhas ocorridas na área da saúde, não são ao acaso e se busca métodos de eliminá-las ou ao menos minimiza-las. Para que isso ocorra é necessário que as instituições hospitalares definam seus processos assistenciais alinhados ao planejamento estratégico da organização e em consonância com o modelo gerencial adotado.

Há de se ponderar os aspectos jurídicos associados aos eventos adversos que tem sido objeto de responsabilidade civil, apontada como causadora de dano a ser indenizado em juízo, pelos responsáveis: hospitais, administração pública e profissionais de saúde (SOUSA P e MENDES W, 2014a).

No âmbito da instituição fonte da pesquisa, encontra-se estabelecido o instrumento "Ficha de Notificação de Eventos Adversos/Queixas Técnicas de Produtos para Saúde". Esse formulário foi elaborado pela assessoria de risco e segurança (ASRS), com vistas a estabelecer a comunicação com os profissionais de saúde para fins de notificação de problemas relacionados ao uso de tecnologias de saúde e processos assistenciais. Sob esse olhar, a pesquisa procurou investigar possíveis aspectos referentes às dificuldades encontradas, pelos profissionais de saúde, para as notificações de incidentes e eventos adversos. Nos resultados obtidos os dados com maior relevância foram quanto à falta de conhecimento sobre a nomenclatura usada para notificar os tipos de eventos adversos, onde se obteve um percentual de $43,7 \%$ de respostas negativas e apenas $16,5 \%$ de afirmativas. Porém, os percentuais encontrados entre as respostas referentes à estrutura dos campos disponibilizados no documento, são significantemente próximos, como demonstra a Tabela 3, apontando para necessidade de melhor estruturação da ficha de notificação utilizada na instituição.

Como enfatizam Paiva MCMS, et al., (2010) os boletins de notificação de eventos adversos, produzidos também em banco de dados, são importantes fontes de aviso e informação, proporcionando segurança no ambiente hospitalar e colaborando para a gestão da assistência.

Embora o sistema de saúde brasileiro tenha desenvolvido ferramentas tecnológicas que estabeleçam meios de comunicação entre as organizações de saúde e órgãos reguladores.

Ainda existem, hospitais brasileiros que são prestadores de serviços e atuam sem avaliar seus processos de trabalho ou usar seus resultados para melhoria continua da qualidade com foco na eficácia e eficiência (CAPUCHO HC e CASSIANI SHB, 2013).

A relevância com que se apresenta o resultado de $72,5 \%$ de profissionais que informaram nunca terem feito alguma notificação de incidentes ou eventos adversos, confirma a inquietação da autora quanto à existência de subnotificações de ocorrências assistenciais.

Nesse aspecto, é preciso considerar a cultura de "culpabilidade individual" adotada pelas organizações de saúde historicamente e incorporada pelos profissionais de saúde, o que acaba favorecendo o receio de dar publicidade as fragilidades percebidas no ambiente de trabalho. 
Para Sousa P e Mendes W (2014a) os erros são consequências e não causas. Destaca a necessidade de incentivar o "pensamento sistêmico" que impulsiona o foco na melhoria do desempenho organizacional e a promoção de uma cultura não punitiva, estimulando o profissional a reconhecer e relatar seus erros. Mas alerta para que a abordagem da "não culpabilidade" não se confunda com as questões que demandam censura e responsabilização.

Nessa perspectiva, torna-se salutar as organizações de saúde que apresentem solidificado o entendimento da gestão de processos, busquem aprimoramentos nos métodos de coletas de dados e monitoramento de maneira estruturada, para assim fortalecer a prática do cuidado, buscando incessantemente a qualidade em saúde, oferecendo melhoria da saúde para os que procuram seus serviços (FONSECA AS, et al. 2014).

\section{CONCLUSÃO}

O estudo permitiu identificar a representatividade da equipe de enfermagem nos processos assistenciais, sendo a participação relevante para os resultados que demonstram quais protocolos estão mais sedimentados nos setores pesquisados. Segundo as referências adotadas, a incorporação dos protocolos institucionais está relacionada a forma como as equipes se organizam no seu ambiente de trabalho e a cultura organizacional. Apontando para necessidade de ações mais efetivas em prol da adesão, pelos profissionais, aos protocolos e os benefícios trazidos para os resultados pretendidos.

\section{AGRADECIMENTOS}

Agradecemos aos servidores da Fundação Hospital de Clínicas Gaspar Viana e da Fundação Santa Casa de Misericórdia do Pará.

\section{REFERÊNCIAS}

1. ANVISA, Agência Nacional de Vigilância Sanitária. Documento de Referência para o Programa Nacional de Segurança do Paciente. Brasília, 2014.

2. BARATA LRB, et al. Hospitais de Ensino e o Sistema Único de Saúde. RAS, v. 12, n. 46, p.7-14, Jan. -mar. 2010.

3. BRASIL. Portaria MS/GM no 529 , de $1^{\circ}$ de abril de 2013. Institui o Programa Nacional de Segurança do Paciente (PNSP). Diário Oficial [da] República Federativa do Brasil, Poder Executivo, Brasília, DF, abr. 2013a.

4. BRASIL. Ministério da Saúde. Documento de Referência para o Programa Nacional de Segurança do Paciente/Ministério da Saúde; Fundação Oswaldo Cruz; Agência Nacional de Vigilância Sanitária. Brasília: 2014a.

5. BROCA PV, FERREIRA MA. Equipe de enfermagem e comunicação: contribuições para o cuidado de enfermagem. REBEn, Brasília, v. 65, n. 1, p. 97-103, Jan. -fev. 2012.

6. CAPUCHO HC, CASSIANI SHB. Necessidade de implementar programa nacional de segurança do paciente no Brasil. Rev Saúde Pública, Brasília, v. 47, n. 4, p. 791-8, ago. 2013.

7. CONSELHO REGIONAL DE ENFERMAGEM DO ESTADO DE SÃO PAULO (COREN-SP). 10 Passos para a segurança do paciente: manual para profissionais. São Paulo, 2010.

8. FISHER ES, et al. Accountable care organizations: accountable for what, to whom, and how. JAMA, v. 304, n. 15, p. 1715-16, 2010.

9. FONSECA AS, et al. Segurança do Paciente. São Paulo: Martinari, 2014.

10. INSTITUTE OF MEDICINE. To err is human. https://www.iom.edu/ /media/Files/Report\%20Files/1999/To-Err-isHuman/To\%20Err\%20is\%20Human\%201999\%20\%20report\%20brief.pdf. 
11. LIMA FDMA Segurança do Paciente e Intervenções para a Qualidade dos Cuidados de Saúde. Revista Espaço para a Saúde. Londrina, v. 15, n. 3, p.22-29, Jul. -set. 2014.

12. MENDES JÚNIOR WV. Torre de Babel. Revista Acreditação; 2(3): 50-57, 2012.

13. NISHIO EA, FRANCO MTG. Modelo de gestão em Enfermagem: qualidade assistencial e segurança do paciente. Rio de Janeiro: Elsevier, 2011.

14. OLIVEIRA JUNIOR R. Eventos adversos notificados ao Sistema Nacional de Notificações para a Vigilância Sanitária (NOTIVISA): Brasil, estudo descritivo no período de 2006 a 2011. Epidemiol. Serv. Saúde, Brasília, v. 22. n. 4, p. 671- 678, out. /dez. 2013.

15. PAIVA MCMS, et al. Eventos adversos: análise de um instrumento de notificação utilizado no gerenciamento de enfermagem. Rev. esc. enferm USP, São Paulo, v. 44, n. 2, p. 287-94, jun. 2010.

16. REBRAENSP (Rede Brasileira de Enfermagem e Segurança do Paciente). Estratégias para a segurança do paciente: manual para profissionais da saúde. Porto Alegre: EDIPUCRS, 2013.

17. SOUSA P, MENDES W. Segurança do Paciente: conhecendo os riscos nas organizações de saúde. v. 1. Rio de Janeiro: EAD/ENSP, 2014a.

18. SOUSA P, MENDES W. Segurança do Paciente: criando organizações de saúde seguras. v. 2. Rio de Janeiro: EAD/ENSP, 2014b.

19. WEMBERG JE. Tracking medicine: a researcher's quest to understand healt care. New York: Oxford University Pres, 2010. 